\title{
Separated before birth: pulsars B2020+28 and B2021+51 as the remnants of runaway stars
}

\author{
V. V. Gvaramadze \\ Sternberg Astronomical Institute, Moscow State University, Universitetskij Pr. 13, Moscow 119992, Russia \\ e-mail: vgvaram@sai.msu.ru \\ Received 23 April 2007 / Accepted 12 May 2007 \\ ABSTRACT

\begin{abstract}
Astrometric data on the pulsars B2020+28 and B2021+51 suggest that they originated within several parsecs of each other in the direction of the Cyg OB2 association. It was proposed that the pulsars share their origin in a common massive binary and were separated at the birth of the second pulsar following the asymmetric supernova explosion. We consider a different scenario for the origin of the pulsar pair based on a possibility that the pulsars were separated before their birth and that they are the remnants of runaway stars ejected (with velocities similar to those of the pulsars) from the core of Cyg OB2 due to strong three- or four-body dynamical encounters. Our scenario does not require any asymmetry in supernova explosions.
\end{abstract}

Key words. pulsars: individual: PSR B2020+28 - open clusters and associations: individual: PSR B2021+51

\section{Introduction}

Most stars form in dense embedded clusters and reside in binary systems (either primordial or tidal). If both binary components are massive enough, they end their lives as core-collapsed supernovae ( $\mathrm{SNe}$ ). Stellar remnants of SN explosions, usually the neutron stars (NSs), have peculiar velocities at least an order of magnitude higher than the typical velocities of their progenitors, the OB stars (e.g. Gunn \& Ostriker 1970). It is believed that the high velocities of NSs are due either to the asymmetry of SN explosions (e.g. Dewey \& Cordes 1987) or to the disruption of tight massive binaries following the second (symmetric) SN explosion (e.g. Iben \& Tutukov 1996). The progress in measuring the proper motions and parallaxes of NSs (pulsars) allows their peculiar (transverse) velocities to be determined with high precision and makes it possible to trace their trajectories back to the parent star clusters (e.g. Hoogerwerf et al. 2001; Chatterjee et al. 2005). Recently Vlemmings et al. (2004; hereafter VCC04) have used the high-precision astrometric data (proper motions and parallaxes) for two dozen pulsars to determine their trajectories in the Galactic potential and to search for pairs with a common origin. They discovered that two pulsars from their sample (presently separated by $\sim 23^{\circ}$ ) originated within several parsecs of each other in the direction of the Cyg OB2 association. VCC04 interpret their discovery as an indication that the progenitors of both pulsars, B2020+28 and B2021+51, were the members of a common massive binary and suggest that the pulsars were separated at the birth of the second one following the asymmetric SN explosion.

In this Letter we explore a different scenario for the origin of B2020+28 and B2021+51. We suggest that these pulsars were separated before their birth and that they are the remnants of runaway stars ejected (with velocities similar to those of the pulsars) from the parent star cluster due to the strong three- or fourbody dynamical encounters. Our scenario does not require any asymmetry in $\mathrm{SN}$ explosions.

\section{Pulsars $B 2020+28$ and $B 2021+51$ : origin in a common binary}

The main result presented in $\mathrm{VCC} 04$ is that $\mathrm{B} 2020+28$ and B2021+51 originated within several parsecs of each other. VCC04 derived the most likely three-dimensional peculiar velocities of the pulsars at birth, $\simeq 150$ and $\simeq 500 \mathrm{~km} \mathrm{~s}^{-1}$ (respectively, for B2021+51 and B2020+28), and the angle between the velocity vectors $\psi \simeq 160^{\circ}$. These velocities can, in principle, be produced via disintegration of a tight (semi-detached) massive binary after the second (symmetric) SN explosion (e.g. Iben \& Tutukov 1996); in this case, however, $\psi$ is always $<90^{\circ}$. Moreover, the spin characteristics of B2020+28 and B2021+51 (typical of non-recycled pulsars) argue against the origin of these pulsars in a common tight binary (cf. VCC04).

One possible way to reconcile the kinematic data with the common binary scenario is to assume that the binary was disrupted either after the first or the second asymmetric SN explosion (VCC04). Note that the similarity between the pulsar's characteristic ages $(\simeq 2.88$ and $\simeq 2.75 \mathrm{Myr})$ implies that the mass ratio of the binary components was $\sim 1$. Therefore, depending on the initial parameters (binary separation, etc), the binary system at the moment of the first SN explosion consists of two red supergiant or Wolf-Rayet stars or of two carbon-oxygen (CO) cores.

The latter situation can be realised if the massive binary evolves through two common-envelope phases (see Belczyński \& Kalogera 2001). A natural outcome of the evolution of this type of binaries, provided that the SN explosions were of zero or moderate asymmetry, is the origin of a binary non-recycled pulsar (Belczyński \& Kalogera 2001). The CO binary, however, could be disrupted after the first (or the second) asymmetric SN explosion if the kick received by the stellar remnant was of proper magnitude and orientation (see Tauris \& Takens 1998).

For illustrative purposes, we consider the disruption of a CO binary following the first asymmetric SN explosion. For parameters of the $\mathrm{CO}$ binary given in 


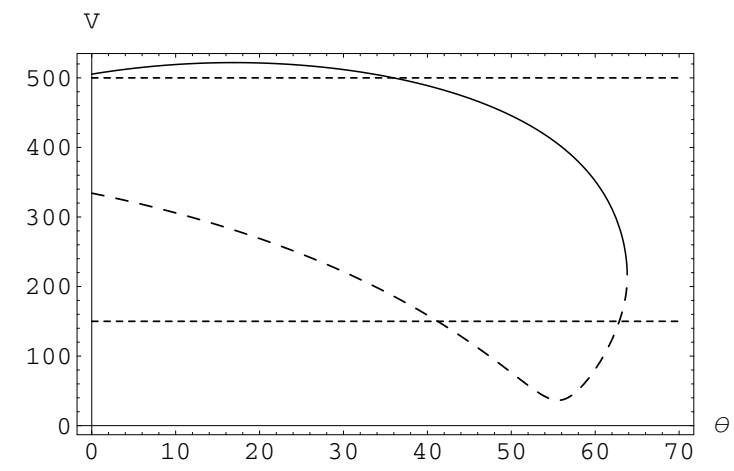

Fig. 1. The dependence of the velocities of the (first-born) pulsar and its former companion star (now the runaway progenitor of the second pulsar) on the angle between the kick vector and the direction of motion of the exploding star (shown, respectively, by the solid and the longdashed lines). The horizontal short-dashed lines indicate the pulsar velocities suggested by VCC04. See text for details.

Belczyński \& Kalogera (2001) and using Eqs. (44)-(47) and (51)-(56) given in Tauris \& Takens (1998), one can show that the pulsar velocities and $\psi$ could be explained if the kick imparted to the first-born pulsar (B2020+28) was $\sim 500 \mathrm{~km} \mathrm{~s}^{-1}$ (for the sake of simplicity we assume that the second SN explosion was symmetric), while the angle between the kick vector and the direction of motion of the exploding star, $\theta$, was $\simeq 40^{\circ}$ (see Figs. 1 and 2 and Gvaramadze 2006). It is obvious that the kick should be stronger if, at the moment of the first SN explosion, the binary consists of red supergiant or Wolf-Rayet stars (cf. VCC04).

Another possibility is that the pulsars attained their velocities in the course of disintegration of the binary after the second asymmetric SN explosion. Since both pulsars are not recycled, one should assume either that the binary separation was sufficiently large (so that the wind of the secondary star did not affect the evolution of the first-born pulsar) or that the binary evolved through a double common-envelope phase (see above). VCC04 suggest that the pulsars were born in a wide binary, but in their analysis they draw an erroneous conclusion that the pulsar velocities can be explained by a kick of only $\simeq 200 \mathrm{~km} \mathrm{~s}^{-1}$ (see Gvaramadze 2006). One can show, however, that in both the above-mentioned cases the kick imparted by the second SN explosion should be $\geq 500 \mathrm{~km} \mathrm{~s}^{-1}$.

Thus, the origin of the pulsars in a common binary implies that at least one of the $\mathrm{SN}$ explosions was asymmetric enough to produce a kick of $\geq 500 \mathrm{~km} \mathrm{~s}^{-1}$. If, however, SNe can indeed impart high velocities to NSs, then it is not necessary to assume that the pulsars originated in the same binary, but instead one can suggest that they were created by two separate SN explosions occurred in the same parent star cluster within a few $10^{5}$ yr. Our scenario for the origin of the pulsar pair has something in common with the latter possibility, but we do not require any asymmetry in the SN explosions.

\section{Pulsars B2020+28 and B2021+51: dynamical ejection from the young massive star cluster}

The recent discovery of the so-called hypervelocity stars (Brown et al. 2005) and hyperfast pulsars (Chatterjee et al. 2005), the ordinary stars and pulsars moving with extremely high $\left(\sim 1000 \mathrm{~km} \mathrm{~s}^{-1}\right)$ peculiar velocities, suggests a possibility that the

\footnotetext{
${ }^{1}$ Note that for $64^{\circ} \lesssim \theta \lesssim 290^{\circ}$, the binary system remains bound.
}

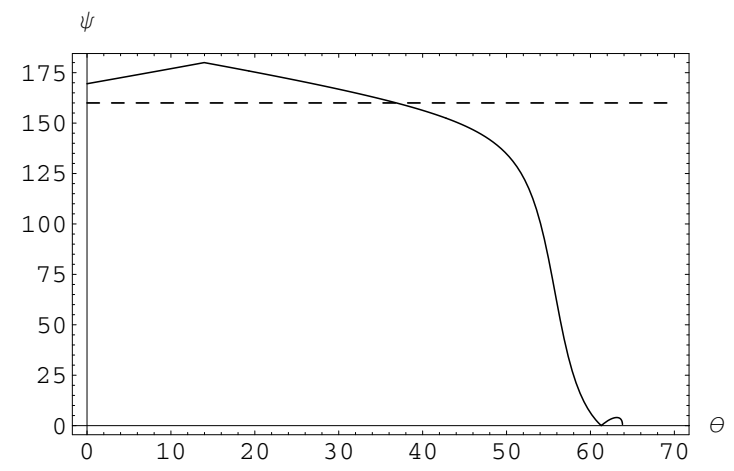

Fig. 2. The angle between the velocity vectors of the first- and secondborn pulsars as a function of the angle between the kick vector and the direction of motion of the exploding star. The horizontal dashed line indicates the angle between the pulsar velocity vectors suggested by VCC04.

hypervelocity stars could be the progenitors of hyperfast NSs, provided that they are massive enough (Gvaramadze et al. 2007). A strong argument in support of this possibility comes from the fact that the mass of one of the hypervelocity stars, HE 0437-5439, is $\gtrsim 8 M_{\odot}$ (Edelmann et al. 2005) so that, in principle, it can end its evolution as a hyperfast NS! The high velocities ( 200-400 $\mathrm{km} \mathrm{s}^{-1}$ ) inferred for some early B-type stars at high galactic latitudes (Ramspeck et al. 2001) also support the possibility that high-velocity pulsars could originate from highvelocity runaway stars.

Gvaramadze et al. (2007) suggest that the origin of hypervelocity stars could be connected not only with scattering processes involving the supermassive black hole $(\mathrm{BH})$ in the Galactic centre (the common wisdom; originally suggested by Hills 1988; see also Yu \& Tremaine 2003; Gualandris et al. 2005), but also with strong three- or four-body dynamical encounters in the dense cores of young massive star clusters (YMSCs), located either in the Galactic disk or near the Galactic centre. The discovery of a halo population of early B stars, whose lifetimes are shorter than the times-of-flight from the Galactic centre (Brown et al. 2007; see also Ramspeck et al. 2001), supports this suggestion. We believe, therefore, that the pulsars B2020+28 and B2021+51 could be the remnants of high-velocity runaway stars ejected from the same YMSC. The kinematic and characteristic ages of the pulsars (respectively, $\sim 2$ and $3 \mathrm{Myr}$; VCC04) imply that by the moment of ejection the progenitor stars have already become Wolf-Rayet stars [the short-lived $(<1 \mathrm{Myr})$ helium $(\mathrm{He})$ cores of massive stars; cf. Gvaramadze et al. 2007].

Of the two mechanisms that could be responsible for the origin of the high-velocity progenitors of B2020+28 and B2021+51, the first relies on close dynamical encounters between hard (Heggie 1975) massive binary stars in the dense core of a YMSC. The peculiar velocities of runaway stars produced in this process are similar to the orbital velocities of the binary components (e.g. Leonard \& Duncan 1990), but occasionally they could be much higher. Scattering experiments by Leonard (1991) showed that the maximum velocity attained by the lightest member of the binaries involved in the interaction (e.g. the He core of a massive star or an early B-type star) can be as high as the escape velocity, $V_{\mathrm{esc}}$, from the surface of the most massive star in the binaries.

For the main-sequence stars with the mass-radius relationship (Habets \& Heintze 1981), $r_{\mathrm{MS}}=0.8\left(m_{\mathrm{MS}} / M_{\odot}\right)^{0.7} R_{\odot}$, where $r_{\mathrm{MS}}$ and $m_{\mathrm{MS}}$ are the stellar radius and the mass, the maximum 
possible velocity of ejected stars is a weak function of $m_{\mathrm{MS}}$, $V_{\mathrm{ej}}^{\max } \simeq V_{\mathrm{esc}} \simeq 700 \mathrm{~km} \mathrm{~s}^{-1}\left(m_{\mathrm{MS}} / M_{\odot}\right)^{0.15}$ and could be as high as $\sim 1400 \mathrm{~km} \mathrm{~s}^{-1}$ (cf. Leonard 1991). Numerical simulations by Leonard (1991) showed that about $4 \%$ of binary-binary encounters result in the origin of runaway stars with $V_{\mathrm{ej}} \simeq 0.5 V_{\mathrm{esc}}$, which is enough to explain the velocity of $\sim 500 \mathrm{~km} \mathrm{~s}^{-1}$ suggested by VCC04 for one of the pulsars. Note that the results of Leonard (1991) were used by Tenjes et al. (2001) to explain the origin of the high-velocity $\left(\sim 400 \mathrm{~km} \mathrm{~s}^{-1}\right)$ runaway star HIP 60350.

Another possible mechanism for producing high-velocity stars is based on exchange encounters between tight binary stars and a compact massive object, either a very massive star (VMS), formed through the runaway stellar collisions and mergers in the mass segregated core of a YMSC (e.g. Portegies Zwart et al. 1999), or its descendant, an intermediate-mass BH (e.g. Portegies Zwart \& McMillan 2002). After the close encounter and tidal breakup of the binary, one of the binary components (usually the more massive one) becomes bound to the compact object, while the second one recoils with a high velocity given by $V_{\mathrm{ej}} \sim\left[M /\left(m_{1}+m_{2}\right)\right]^{1 / 6}\left(2 G m_{1} / a\right)^{1 / 2}$ (Hills 1988; see also Gvaramadze et al. 2007), where $M$ is the mass of the compact object, $m_{1}$ and $m_{2}$ are the masses of the binary components $\left(m_{1}>m_{2}\right)$, and $a$ the binary separation.

In YMSCs of mass $\sim 10^{4} M_{\odot}$, the mass of the VMS does not exceed several $100 M_{\odot}$, while the thermal (Kelvin-Helmholtz) time scale of the VMS is shorter than the mean time between collisions (see Portegies Zwart et al. 1999). In this case, the growing VMS rapidly evolves to the thermal equilibrium (e.g. Suzuki et al. 2007), so that one can adopt the following massradius relationship, $R \simeq 1.6\left(M / M_{\odot}\right)^{0.47} R_{\odot}$, where $R$ is the radius of the VMS (see Freitag et al. 2006, and references therein). In the process of an exchange encounter with a binary, the VMS could be considered as a point mass if the binary tidal radius, $r_{\mathrm{t}} \sim\left[M /\left(m_{1}+m_{2}\right)\right]^{1 / 3} a$, is at least several times larger than $R$. For $M=200-300 M_{\odot}, m_{1}=30 M_{\odot}$ (a main-sequence star), $m_{2}=$ $8 M_{\odot}$ (a He core), and $a=50 R_{\odot}$, one has $r_{\mathrm{t}} \simeq 90-100 R_{\odot}$ (i.e. much larger than $R \simeq 19-23 R_{\odot}$ ) and $V_{\text {ej }} \simeq 630-670 \mathrm{~km} \mathrm{~s}^{-1}$, that is enough to explain the pulsar velocities.

In more massive $\left(\geq 10^{5} M_{\odot}\right)$ YMSCs, the VMS can acquire a mass of several $1000 M_{\odot}$ (Portegies Zwart et al. 2004). But in this case, the thermal time scale is comparable to the collision time and the VMS remains inflated untill collapsing into an intermediate-mass BH (e.g. Portegies Zwart et al. 2006). Exchange encounters with this VMS would not produce high ejection velocities. The star ejection from the YMSC, however, would be very effective if the VMS leave behind a BH of mass $\sim 1000 M_{\odot}$ (e.g. Gualandris \& Portegies Zwart 2007).

\section{Cyg OB2}

The astrometric data on B2020+28 and B2021+51 suggest that these pulsars (or their progenitors; our preference) were ejected $\sim 1.9$ Myr ago from the same origin at a distance of $\sim 1.9 \mathrm{kpc}$ in the direction of the Cyg OB2 association (VCC04). The parent YMSC (or its descendant) should still be located at about the same distance since its possible peculiar velocity of $\leq 30 \mathrm{~km} \mathrm{~s}^{-1}$ (typical of the OB associations near the Sun; de Zeeuw et al. 1999) would result only in a slight offset of $\leq 60 \mathrm{pc}$ (cf. VCC04). To constrain the current age of the parent cluster, we assume that the initial mass of the progenitor stars of B2020+28 and B2021+51 could be as high as $\gtrsim 50 M_{\odot}$. (It is believed that stars of this mass can lose most of their mass via stellar wind or mass transfer on a binary companion and leave behind NSs; e.g. Vanbeveren et al. 1998; Wellstein \& Langer 1999; cf. Muno et al. 2006.) From this it follows that the minimum age of the parent YMSC should be $\sim 5 \mathrm{Myr}$, that is, $\sim 2 \mathrm{Myr}$ (the pulsar kinematic age) plus $\sim 3 \mathrm{Myr}$ (the lifetime of a $\gtrsim 50 M_{\odot}$ star). Assuming that the YMSC initially contained at least 10 stars of mass $>50 M_{\odot}$, one has the (initial) mass of the cluster of $\geq 10^{4} M_{\odot}$ (for a $0.2-120 M_{\odot}$ Salpeter initial mass function).

The only likely candidate for the birth cluster of B2020+28 and $\mathrm{B} 2021+51$ in the region suggested by $\mathrm{VCC} 04$ is the Cyg OB2 association. Numerous star clusters in its neighbourhood (see, e.g., Le Duigou \& Knödlseder 2002) cannot pretend to play this role either due to their youth or low masses.

Cyg OB2 is one of the most massive and compact OB associations in our Galaxy (Knödlseder 2000). The large number ( 100) of O stars identified in Cyg OB2 (Knödlseder 2000; see also Comerón et al. 2002) implies that its mass could be as high as $\sim 10^{5} M_{\odot}$. The angular radius of Cyg OB2 is $\sim 1^{\circ}$, while the half light radius is $\sim 13^{\prime}$ (Knödlseder 2000), that at the distance of Cyg OB2 of 1.5-1.7 kpc (Hanson 2003; Massey \& Thompson 1991) corresponds, respectively, to $\sim 25-30 \mathrm{pc}$ and $~ 5-6$ pc. Note that the centre of Cyg OB2 lies within the $2 \sigma$ likelihood contour of the pulsar birth location and, at the $2 \sigma$ level, the distances to the Cyg OB2 and the birth location are consistent with each other. Age estimates for Cyg OB2 range from $\sim 1$ to 5 Myr (e.g. Bochkarev \& Sitnik 1985; Knödlseder et al. 2002). The wide age spread suggests that the star formation in the Cyg OB2 was non-coeval. The non-coevality could be understood if the star formation in the association started initially in the dense core of the parent molecular cloud and then propagated to its lower density periphery. It is believed (e.g. Elmegreen 2000) that the star formation occurs on one or two dynamical time scales, $t_{\text {dyn }} \sim(G \rho)^{-1 / 2}$, where $\rho$ is the gas density in the cloud, so that in a density-stratified cloud of mass of $\sim 10$ times higher than the stellar mass of Cyg OB2 and the size similar to that of the association, the age spread could be comparable with $t_{\text {dyn }} \sim 5$ Myr.

If the progenitor stars of B2020+28 and B2021+51 were ejected from Cyg OB2, then we suppose that, 2 Myr ago (or $\sim 3 \mathrm{Myr}$ after the formation of the first massive stars in the centre of the association), the core of the association was much more compact and denser. Assuming that the association expands with a velocity equal to its velocity dispersion $\left(\simeq 2.4 \mathrm{~km} \mathrm{~s}^{-1}\right.$; Kiminki et al. 2006), one finds that the stars located within the current half light radius were originally concentrated in a region of a radius of $<1 \mathrm{pc}$. It is likely that the two star clusters projected close to each other $\left(\sim 6^{\prime}\right)$ near the centre of Cyg OB2 (Bica et al. 2003) are the remainders of this dense core. We suggest that the mass of the core was much higher $\left(\geq 10^{4} M_{\odot}\right)$ than the current mass of the clusters (several $1000 M_{\odot}$; Bica et al. 2003) and that it was significantly reduced during the last $2 \mathrm{Myr}$ due to the overall expansion of the association and star ejections following close dynamical encounters (recent $N$-body simulations by Pflamm-Altenburg \& Kroupa (2006) showed that dynamical processes in the Trapezium cluster could be responsible for the loss of at least $75 \%$ of its initial content of OB stars). Thus we believe that $\sim 2 \mathrm{Myr}$ ago the conditions in the core of Cyg OB2 were favourable to the dynamical processes discussed in Sect. 3.

Acknowledgements. I am grateful to H. Baumgardt, D. Bomans, P. Kroupa, and S. Portegies Zwart for useful discussions. I am also grateful to D. Bomans and R.-J. Dettmar for their hospitality during my stay at the Astronomisches Institut, Ruhr-Universität Bochum, where this work was partially carried out. This work was partially supported by the Deutsche Forschungsgemeinschaft. 


\section{References}

Belczyński, K., \& Kalogera, V. 2001, ApJ, 550, L183

Bica, E., Bonatto, Ch., \& Dutra, C. M. 2003, A\&A, 405, 991

Bochkarev, N. G., \& Sitnik, T. G. 1985, ApSS, 108, 237

Brown, W. R., Geller, M. J., Kenyon, S. J., \& Kurtz, M. J. 2005, ApJ, 622, L33

Brown, W. R., Geller, M. J., Kenyon, S. J., Kurtz, M. J., \& Bromley, B. C. 2007, [arXiv:astro-ph/0701600]

Chatterjee, S., Vlemmings, W. H. T., Brisken, W. F., et al. 2005, ApJ, 630, L61

Comerón, F., Pasquali, A., Rodighiero, G., et al. 2002, A\&A, 389, 874

de Zeeuw, P. T., Hoogerwerf, R., de Bruijne, J. H. J., Brown, A. G. A., \& Blaauw, A. 1999, AJ, 117,354

Dewey, R. J., \& Cordes, J. M. 1987, ApJ, 321, 780

Edelmann, H., Napiwotzki, R., Heber, U., Christlieb, N., \& Reimers, D. 2005, ApJ, 634, L181

Elmegreen, B. G. 2000, ApJ, 530, 277

Freitag, M., Rasio, F. A., \& Baumgardt, H. 2006, MNRAS, 368, 121

Gualandris, A., \& Portegies Zwart, S. 2007, MNRAS, 376, L29

Gualandris, A., Portegies Zwart, S., \& Sipior, M. S. 2005, MNRAS, 363, 223

Gunn, J. E., \& Ostriker, J. P. 1970, ApJ, 160, 979

Gvaramadze, V. V. 2006, A\&A, 454, 239

Gvaramadze, V. V., Gualandris, A., \& Portegies Zwart, S. 2007, [arXiv:astro-ph/0702735]

Hanson, M. M. 2003, ApJ, 597, 957

Habets, G. M. H. J., \& Heintze, J. R. W. 1981, A\&AS, 46, 193

Heggie, D. C. 1975, MNRAS, 173, 729

Hills, J. G. 1988, Nature, 331, 687

Hoogerwerf, R., de Bruijne, J. H. J., \& Zeeuw, P. T. 2001, A\&A, 365, 49
Iben, I., Jr., \& Tutukov, A. V. 1996, ApJ, 456, 738

Kiminki, D. C., Kobulnicky, H. A., Kinemuchi, K., et al. 2006 , [arXiv: astro-ph/0609772]

Knödlseder, J. 2000, A\&A, 360, 539

Knödlseder, J., Cerviño, M., Le Duigou, J.-M., et al. 2002, A\&A, 390, 945

Le Duigou, J.-M., \& Knödlseder, J. 2002, A\&A, 392, 869

Leonard, P. J. T. 1991, AJ, 101, 562

Leonard, P. J. T., \& Duncan, M. J. 1990, AJ, 99, 608

Massey, P., \& Thompson, A. B. 1991, AJ, 101, 1408

Muno, M. P., Clark, J. S., Crowther, P. A., et al. 2006, ApJ, 636, L41

Pflamm-Altenburg, J., \& Kroupa, P. 2006, MNRAS, 373, 295

Portegies Zwart, S. F., \& McMillan, S. L. W. 2002, ApJ, 576, 899

Portegies Zwart, S. F., Makino, J., McMillan, S. L. W., \& Hut, P. 1999, A\&A, 348,117

Portegies Zwart, S. F., Baumgardt, H., Hut, P., Makino, J., \& McMillan, S. L. W. 2004, Nature, 428, 724

Portegies Zwart, S. F., Baumgardt, H., McMillan, S. L. W., et al. 2006, ApJ, 641, 319

Ramspeck, M., Heber, U., \& Moehler, S. 2001, A\&A, 378, 907

Suzuki, T. K., Nakato, N., Baumgardt, H., et al. 2007, ApJ, submitted, [arXiv: astro-ph/0703290]

Tauris, T. M., \& Takens, R. J. 1998, A\&A, 330, 1047

Tenjes, P., Einasto, J., Maitzen, H. M., \& Zinnecker, H. 2001, A\&A, 369, 530

Vanbeveren, D., Van Rensbergen, W., \& De Loore 1998, The Brightest Binaries (Boston: Kluwer Academic Publishers)

Vlemmings, W. H. T., Cordes, J. M., \& Chatterjee, S. 2004, ApJ, 610, 402

Wellstein, S., \& Langer, N. 1999, A\&A, 350, 148

Yu, Q., \& Tremaine, S. 2003, ApJ, 599, 1129 